\title{
EXAMPLE OF A NONACYCLIC CONTINUUM SEMIGROUP $S$ WITH ZERO AND $S=E S E$
}

\author{
ANNE L. HUDSON ${ }^{1}$
}

Throughout this discussion $S$ will denote a compact connected topological semigroup and $E$ will denote the set of idempotents of $S$. The problem to be considered concerns a question posed by Professor A. D. Wallace. In [1], Wallace proves that if $S$ has a left unit, if $I$ is a closed ideal of $S$, and if $L=\square$ or if $L$ is a closed left ideal of $S$, then $H^{n}(S) \cong H^{n}(I \cup L)$ for all integers $n$, where $H^{n}(A)$ denotes the $n$th Alexander-Cech cohomology group of $A$ with coefficients in an arbitrary but fixed group $G$. If $S$ is assumed to have both a left zero and a left unit, then it follows that each closed left ideal $L$, of $S$ is acyclic; that is, $H^{p}(L)=0$ for all $p \geqq 1$. A dual statement holds for closed right ideals if $S$ has a right unit and right zero. A generalization of the case in which $S$ has a left, right, or two-sided unit, is to require that $S=E S, S=S E$, or $S=E S E$, respectively, and Wallace has asked: "If $S$ has a zero, are closed right or left ideals of $S$ necessarily acyclic in the more general situation?" [3]. A negative answer to this question is given here by way of examples, and a theorem is proved giving a necessary and sufficient condition for closed right ideals of $S$ to be acyclic, assuming $S=E S E$ and $S$ has a zero. Following the proof of this theorem is an example of a semigroup not satisfying this condition.

The above-mentioned example shows that even though $S$ is acyclic, it is not necessarily true that all closed right ideals of $S$ are acyclic. Thus the question remains as to whether $S$ is acyclic if $S=E S E$ and $S$ has a zero [3]. Wallace proves in [2] that for such a semigroup $S$, $H^{1}(S)=0$, however, an example is given here of a semigroup $S$ with zero, $S=E S E$ and $H^{2}(S) \cong G$ for all groups $G$, showing that this question also has a negative answer. Two further examples are included in this paper which show what can occur if one only assumes that $S=S E$, or $S=E S$. One example is of a semigroup $S$ with zero, $S=S E$ and $H^{1}(S) \cong G$ for all groups $G$ and the other is an example of a semigroup $S$ with zero and left unit and $S$ contains a closed right ideal $R$ with $H^{1}(R) \cong G$.

Definition. Let $T$ be a semigroup, $a \in T$ and $R(a)$ the closed right ideal of $T$ generated by $a$. Then $a$ is said to be right codependent on

Received by the editors May 2, 1962.

1 This paper was prepared while the author was the holder of a National Science Foundation Postdoctoral fellowship. 
$T$ if for any integer $n \geqq 1, H^{n}(T)=0$ implies that $H^{n}(R(a))=0$.

THEOREM. Let $S$ be a compact connected semigroup with zero and $S=E S E . A$ necessary and sufficient condition that each closed right ideal of $S$ be acyclic is that each $a$ in $S$ be right codependent on $S$.

The proof of this theorem depends on the following two lemmas. The proofs of these lemmas are paraphrases of the proof of the main theorem in [2] and will be omitted.

LeMma 1. Let $S$ be a compact connected semigroup with zero and $S=E S$. Let $n$ be a fixed integer $n \geqq 2$. If $H^{n-1}(R)=0$ for each closed right ideal $R \subset S$, then $H^{n}(S)=0$.

LEMma 2. Let $S$ be a compact connected semigroup with zero and $S=S E$. Let $n$ be a fixed integer, $n \geqq 1$. If for each $a \in S, H^{n}(a S)=0$ and if for each closed subset $A \subset S, H^{n-1}(A S)=0$, then $H^{n}(R)=0$ for each closed right ideal $R \subset S$. (For $n=1$, reduced groups are to be used.)

Proof of Theorem. First assume that each $a$ in $S$ is right codependent on $S$. The proof of sufficiency will be by induction on $n$. Let $n=1$. From [2], $H^{1}(S)=0$, hence it follows that $H^{1}(a S)=0$ for each $a$ in $S$ since $R(a)=a S$ and each $a$ is right codependent on $S$. Each closed right ideal $R \subset S$ is connected, therefore $H^{0}(R, r)=0$ for each $r \in R$. Thus, using reduced groups, it follows from Lemma 2 that $H^{1}(R)=0$ for each closed right ideal $R \subset S$.

Assume now that $H^{k-1}(R)=0$ for each closed right ideal $R$ of $S$ and integer $k \geqq 2$. Then by Lemma $1, H^{k}(S)=0$, hence $H^{k}(a S)=0$ for each $a \in S$. Applying Lemma 2, it follows that $H^{k}(R)=0$ where $R$ is a closed right ideal of $S$. This completes the proof of sufficiency.

If each closed right ideal of $S$ is acyclic, then $H^{n}(a S)=0$ for each $a \in S$, integer $n \geqq 1$ and coefficient group $G$ since $a S$ is a closed right ideal. Also $R(a)=a S$ for each $a \in S$ so that it is trivially true that each $a$ in $S$ is right codependent on $S$ which completes the proof of the theorem.

In the following examples, let $I=[0,1]$ denote the real unit interval and for $x$ and $y$ in $I$ let:

$$
\begin{aligned}
x \wedge y & =\text { minimum of } x \text { and } y, \\
x \vee y & =\text { maximum of } x \text { and } y, \\
x y & =\text { real product of } x \text { and } y .
\end{aligned}
$$

EXAMPLE 1. This is an example of a compact connected semigroup $S$ with zero, $S=E S E, S$ is acyclic and there exists an element $p$ in $S$ with $H^{1}(p S) \cong G$ for all groups $G$. This example shows that there exist 
semigroups with zero such that each element is not right codependent on $S$. The topological space of $S$ is a two-cell with three closed intervals, $I_{1}, I_{2}$, and $I_{3}$, issuing from a common point $z_{0}$, on the boundary, $B$, of the two-cell. This point $z_{0}$ is the zero of $S$ and $p \in B \backslash z_{0}$. By construction $p S=B$ and $B^{2}=z_{0}$. In this example, $E=\left\{e_{1}, e_{2}, e_{3}, z_{0}\right\}$ where $e_{i}$ is the free endpoint of $I_{i}$.

Example 1 is constructed as follows. Let $\{a, b, c, d, \theta\}$ be a discrete space consisting of five elements. Define spaces $A, B, C, D$ and $S_{0}$ as follows:

$A=a \times I, B=b \times I, C=c \times I, D=d \times I \times I$, each with the product topology and $S_{0}=A \cup B \cup C \cup D \cup\{\theta\}$ with the topology on $S_{0}$ given by the union of the topologies on $A, B, C, D$ and $\{\theta\}$. Define the product $p q$ for $p$ and $q$ in $S_{0}$ by:

$$
p q=\left\{\begin{array}{l}
(d,(x \wedge y) r,(x \vee y)), \text { if } p=(d, x, y) \in D, q=(a, r) \in A, \\
(d,(x \vee y),(x \wedge y) r), \text { if } p=(d, x, y) \in D, q=(b, r) \in B, \\
(b, r s), \text { if } p=(a, r) \in A, \text { or } p=(b, r) \in B \text { and } q=(b, s) \in B, \\
(a, r s), \text { if } p=(b, r) \in B, \text { or } p=(a, r) \in A \text { and } q=(a, s) \in A, \\
(d, x r, y r), \text { if } p=(c, r) \in C \text { and } q=(d, x, y) \in D, \\
(c, r s), \text { if } p=(c, r) \in C \text { and } q=(c, s) \in C, \\
\theta \text { otherwise. }
\end{array}\right.
$$

By the definition of the topology on $S_{0}$, multiplication is continuous and associativity is checked by direct computation. Let $E_{0}=$ $\{(a, 1),(b, 1),(c, 1), \theta\}$. Then $E_{0}$ is a set of idempotents in $S_{0}$ and the claim is made that $S_{0}=E_{0} S_{0} E_{0}$. This is true since $(a, 1)$ is a twosided unit for $A$ and a right unit for $(d \times\{(x, y): x \leqq y\}) ;(b, 1)$ is a two-sided unit for $B$ and a right unit for $(d \times\{(x, y): x \geqq y\}) ;(c, 1)$ is a two-sided unit for $C$ and a left unit for $D$; and $\theta^{2}=\theta$.

Consider now, $I_{0}=(d \times\{0\} \times I) \cup(d \times I \times\{0\}) \cup\{(a, 0),(b, 0)$, $(c, 0), \theta\}$. By direct computation it can be shown that this closed subset of $S_{0}$ is a two-sided ideal of $S_{0}$. Let $S=S_{0} / I_{0}$ be the Rees quotient of $S_{0}$ by $I_{0}$. Then $S$ is a compact connected semigroup with zero and it is clear that $S$ is acyclic. Also the condition $S_{0}=E_{0} S_{0} E_{0}$ implies that $S=E S E$ where $E$ is the set of idempotents of $S$.

Let $p=(d, 1,1)$. Then $p S=\left((d, 1,1) S_{0} \cup I_{0}\right) / I_{0}=((d \times I \times\{1\})$ $\left.\cup(d \times\{1\} \times I) \cup I_{0}\right) / I_{0}$ so that $p S$ is homeomorphic to a one-sphere and therefore $H^{1}(p S) \cong G$ for all groups $G$.

EXAMPLE 2. This is an example of a compact connected semigroup $S$ with zero, $S=E S E$ and $H^{2}(S) \cong G$ for all coefficient groups $G$. The topological space of this semigroup is a two-sphere with four closed intervals, $I_{i}, i=1,2,3,4$, issuing from a common point, $z_{1}$, on the two- 
sphere. The point $z_{1}$ is a zero for $S$ and if $e_{i}$ denotes the free endpoint of $I_{i}$, then $E=\left\{e_{1}, e_{2}, e_{3}, e_{4}, z_{1}\right\}$ and multiplication in $S$ has the following properties: (Let $S_{1}$ denote the two-sphere in $S ; C_{1}$ and $C_{2}$ the two great circles in $S_{1}$ through $z_{1} ; H_{1}$ and $H_{2}$ the closed hemispheres determined by $C_{1}$; and $P_{1}, P_{2}$ the closed hemispheres determined by $C_{2}$.) $S_{1}^{2}=z_{1} ; \quad e_{1} S=H_{1} \cup I_{1} ; \quad e_{2} S=H_{2} \cup I_{2} ; S e_{3}=P_{1} \cup I_{3} ; S e_{4}=P_{2} \cup I_{4}$. Hence $e_{1} S \cap e_{2} S=C_{1}$ is a closed right ideal of $S$ with nontrivial cohomology in dimension one. Similarly, $\mathrm{Se}_{3} \cap \mathrm{Se}_{4}=C_{2}$ is a closed left ideal of $S$.

$S$ is constructed in the following way: Let $N=\{a, b, c, d, e, \theta\}$ be a discrete space with six elements. Let $N_{0}=N \backslash\{e, \theta\}$ and let $T=\left(N_{0} \times I\right) \cup(e \times I \times I) \cup\{\theta\}$ with the topology on $T$ given by the union of the topologies of its subsets. For $x$ and $y$ in $[0,1]$ define $\alpha(x, y)=(x \wedge y \wedge(1-x) \wedge(1-y))$ and define the product $p q$ for $p$ and $q$ in $T$ by:

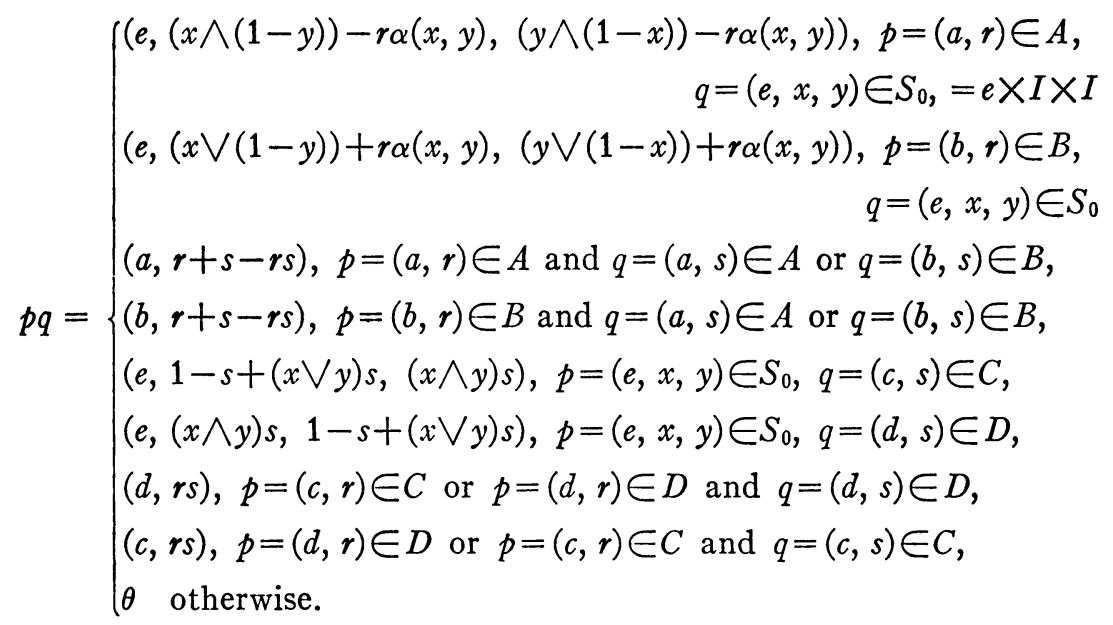

By the definition of the topology on $T$, it is clear that multiplication is continuous since it involves continuous operations of real numbers. By direct computation it is seen that multiplication is also associative and therefore $T$ is a compact semigroup. Let $S_{i}, i=1,2,3,4$, subsets of $e \times I \times I$ be defined by:

$$
\begin{aligned}
& S_{1}=\{(e, x, y): 0 \leqq x \leqq y \leqq x+y \leqq 1\}, \\
& S_{2}=\{(e, x, y): 0 \leqq x \leqq y \leqq 1 \leqq x+y\}, \\
& S_{3}=\{(e, x, y): 0 \leqq y \leqq x \leqq 1 \leqq x+y\}, \\
& S_{4}=\{(e, x, y): 0 \leqq y \leqq x \leqq x+y \leqq 1\},
\end{aligned}
$$

and let $E_{0}=\{(a, 0),(b, 0),(c, 1),(d, 1), \theta\} . E_{0}$ is a set of idempotents 
in $T$ and the claim is made that $T=E_{0} T E_{0}$. This follows from the following equalities:

$a \times I=(a, 0)(a \times I)(a, 0) ; b \times I=(b, 0)(b \times I)(b, 0) ; c \times I$ $=(c, 1)(c \times I)(c, 1) ; d \times I=(d, 1)(d \times I)(d, 1) ; \theta^{2}=\theta$ and $e \times I \times I$ $=\bigcup\left\{S_{i}: i=1,2,3,4\right\}=(a, 0) S_{1}(d, 1) \cup(b, 0) S_{2}(d, 1) \cup(b, 0) S_{3}(c, 1)$ $\cup(a, 0) S_{4}(c, 1)$. This proves that $T=E_{0} T E_{0}$ as claimed.

Now let $I_{0}=\{(a, 1),(b, 1),(c, 0),(d, 0), \theta\} \cup(e \times F(I \times I))$ where $F(I \times I)$ denotes the boundary of $I \times I$ in the Euclidean plane. It can be shown that this closed subset of $T$ is a two-sided ideal of $T$, hence $S=T / I_{0}$ is a compact connected semigroup as described above. Also $S=E S E$, since $T=E_{0} T E_{0}$ and $S$ has a zero.

EXAMPLE 3. This example is of a semigroup $S=S E$ which is compact connected, has a zero and $H^{1}(S) \cong G$ for all groups $G$. $S$ is a subsemigroup of the semigroup in Example 1 and the topological space of $S$ is a circle with two closed intervals issuing from a common point of the circle.

In the terminology of Example 1, consider the following closed subsemigroup, $T$, of $S_{0}$ :

$T=A \cup B \cup(d \times F(I \times I)) \cup\{\theta\}$. Then $T=T(a, 1) \cup T(b, 1) \cup\{\theta\}$ so that $T=T E_{1}$ where $E_{1}$ is the set of idempotents in $T$. Let $I_{1}$ $=\{(a, 0),(b, 0), \theta\} \cup(d \times\{0\} \times I) \cup(d \times I \times\{0\})$. Then $I_{1}=T \cap I_{0}$ is a closed ideal of $T$ and $S=T / I_{1}$ is a compact connected semigroup with zero and $S=S E$. Clearly $S$ is topologically as described above, so that $H^{1}(S) \cong G$.

ExAmple 4. This final example is of a semigroup $S$ with zero and left unit and $S$ contains a closed right ideal $R$ such that $H^{1}(R) \cong G$, for all groups $G$.

Let $S=(\{0\} \times I \times I) \cup(I \times\{0\} \times I)$ and define multiplication in $S$ by $(x, y, z)(r, s, t)=(x r, x s, z t)$. $S$ can be represented by the following matrix semigroup:

$$
\left\{\left(\begin{array}{lll}
x & y & 0 \\
0 & 0 & 0 \\
0 & 0 & z
\end{array}\right):(x, y, z) \in S\right\}
$$

so that multiplication in $S$ is continuous and associative. Clearly $(0,0,0)$ is a zero for $S$ and $(1,0,1)$ is a left unit for $S$. By the definition of multiplication it follows that any subset of $(\{0\} \times I \times I)$ containing $(\{0\} \times\{0\} \times I)$ is a right ideal of $S$ and, in particular, $R=$ the boundary of $(\{0\} \times I \times I)$ is a closed right ideal of $S$ and $H^{1}(R) \cong G$ for all coefficient groups $G$.

In these four examples it might be noted that the set of idem- 
potents in each semigroup was a finite discrete set. It might be of interest to know if there exists a semigroup $S=E S E$ which is compact connected, has a zero, is not acyclic and such that the set of idempotents is connected.

\section{BIBLIOGRAPHY}

1. A. D. Wallace, Acyclicity of compact connected semigroups, Fund. Math. 1 (1961), 99-105.

2. - Cohomology, dimension and mobs, Summa Brasil. Math. 3 (1953), 43-55.

3. - - Problems on semigroups, Colloq. Math. 8 (1961), 223-224.

TUlane University

\section{CONFORMAL VECTOR FIELDS IN COMPACT RIEMANNIAN MANIFOLDS}

T. K. PAN

1. Introduction. Let $V^{n}$ be a compact Riemannian manifold of dimension $n$ and of class $C^{3}$. Let $g_{i j}(x)$ of class $C^{2}$ be the coefficients of the fundamental metric which is assumed to be positive definite. Let $\Gamma_{i j}^{h}$ be the Christoffell symbol, $R_{i j h k}$ the curvature tensor and $R_{i j}$ the Ricci tensor.

Let $\phi$ be an arbitrary scalar invariant, $\xi^{i}$ an arbitrary vector field and $\xi_{i_{1} i_{2}} \ldots i_{p}$ an arbitrary anti-symmetric tensor field of order $p$, all of class $C^{2}$ in $V^{n}$. We shall make use of the following results obtained by S. Bochner and K. Yano [1, pp. 31, 51, 69]:

(1.1) $\left(\Delta \phi \geqq 0\right.$ everywhere in $\left.V^{n}\right) \Rightarrow\left(\phi=\right.$ constant everywhere in $\left.V^{n}\right)$.

$$
\int_{V^{n}} \xi^{i}, i d v=0
$$

$$
\begin{gathered}
\int_{V^{n}}\left(R_{i j} \xi^{i} \xi^{j}+\xi^{i},_{j} \xi^{j},{ }_{i}-\xi^{i},{ }_{i} \xi^{j},{ }_{j}\right) d v=0 . \\
\int_{V^{n}}\left(F\left\{\xi_{i_{1} i_{2} \cdots i_{p}}\right\}+\xi^{i i_{2} \cdots i_{p}, j} \xi_{j i_{2} \cdots i_{p}, i}-\xi^{i i_{2} \cdots i_{p},{ }_{i} \xi^{j}{ }_{i_{2}} \cdots i_{p}, j}\right) d v=0
\end{gathered}
$$

where

Presented to the Society, August 29, 1961; received by the editors January 2, 1962 and, in revised form, May 10, 1962. 